\title{
Superconducting cavity material for the European XFEL
}

\author{
W. Singer, X. Singer, A. Brinkmann, J. Iversen, A. Matheisen, A. Navitski, \\ DESY, Hamburg, Germany \\ Y. Tamashevich, University of Hamburg, Germany \\ P. Michelato, L. Monaco, INFN Milano-LASA, Segrate, (Mi), Italy
}

\begin{abstract}
Analysis of the strategy for superconducting cavity material procurement and quality management is done on the basis of the experience with the cavity production for the European X-ray Free Electron Laser (EXFEL) facility. An adjustment of the material specification to EXFEL requirements, procurement of material, quality control (QC), documentation, and shipment to cavity-producers have been worked- and carried out by DESY. A multistep process of qualification of the material suppliers included detailed material testing, single- and nine-cell cavity fabrication and cryogenic radiofrequency tests. Production of about 25,000 semi-finished parts of high purity niobium and niobium-titanium alloy in a period of three years has been divided finally between companies Heraeus, Tokyo Denkai, Ningxia OTIC, and PLANSEE. Large-grain (LG) material as a possible option for the EXFEL has been considered and resulted in the production of one cryogenic module consisting of seven (out of eight) LG cavities. They fulfilled the EXFEL requirements and showed even 25 to $30 \%$ higher unloaded quality factor. A possible shortage of the required quantity of LG material on the market leaded, however, to the choice of conventional fine grain material. The eddy-current scanning has been applied as an additional QC tool for the niobium sheets and contributed significantly to the material qualification and sorting. $2 \%$ of the sheets have been rejected what potentially could affect up to $1 / 3$ of the cavities. The main imperfections and defects in the rejected sheets have been analyzed. Samples containing foreign material inclusions have been extracted from the sheets and electrochemically polished. Some inclusions remained even after $150 \mu \mathrm{m}$ surface layer removal. Indications of foreign material inclusions have been found in the industrially fabricated and treated cavities and a deeper analysis of the defects has been performed.
\end{abstract}

\section{Introduction}

Superconducting radio-frequency (SRF) cavities are a key component of current and future efficient particle accelerators producing high-energy and high-intensity beams [1, 2]. Utilization of SRF cavities for particle accelerators is steadily growing during the last three decades. Material for superconducting cavities should generally satisfy two main requirements: on one hand provide the RF performance and on the other hand should have properties ensuring the mechanical production and stability of the shape. The SRF cavities are usually made of a high-purity niobium and undergo a complex multistep production process to achieve high accelerating gradient $E_{a c c}$ and unloaded quality factor $Q_{0}$. Niobium was proposed as material for superconducting accelerating cavities at Stanford University. Having the highest critical temperature and critical magnetic field of all pure metals it is still an indisputable preferred metal for the fabrication of superconducting cavities [3-5]. Niobium is chemically inert (at room temperature, the surface is covered by a protecting pentoxide layer), it can be easily machined and deep drawn, and it is available on the market, in the required amounts, in bulk and sheet material forms. Though the options, competing with bulk niobium, demonstrated evident progress in the last years (for example $\mathrm{Nb}_{3} \mathrm{Sn}$ [6], energetic thin films deposition $[7,8]$, multilayers $[9,10])$, nevertheless they still cannot be considered as a mature technique for a series cavity production. Therefore, the most of the next accelerator projects (e.g. International Linear Collider (ILC) [11], European Spallation Source (ESS) [12], and Linac Coherent Light Source II" (LCLS-II) [13]) rely on the SRF cavities fabrication, planning using cavities from bulk high purity niobium.

Recently developed treatment based on nitrogen doping gives a new swing to niobium [14, 15]. This procedure allows up to a factor of three increase of the quality factor of SRF cavities and has an important outcome for accelerators like LCLS-II operating in continuous wave $(\mathrm{CW})$ mode.

It was not a surprise that the bulk high purity niobium has been chosen as a material for the superconducting cavities of the European X-ray Free Electron Laser (EXFEL) facility [16]. The EXFEL requires 800 TESLA-shape [17] nine-cell $1.3 \mathrm{GHz}$ SRF niobium cavities operating at nominal average $E_{a c c}$ of 23.6 $\mathrm{MV} / \mathrm{m}$ with $Q_{0}$ of at least $10^{10}$.

Approximately 20 tons of high purity niobium was required for twelve different types of semi-finished products (SFP) for the cavities of the EXFEL. This is the largest serial production in the history of SRF cavities. Moreover, the material had to be produced according to pressure directive requirements [18].

Another aspect which has to be considered for the procurement is the type of material, i.e. fine grain (FG) or large grain (LG) niobium. LG material (called also ingot 
niobium) allows an elimination of the long production chain from ingot to niobium sheet and has a number of benefits [19]. A special program investigating the advantages and disadvantages of LG was set up for the EXFEL at DESY.

The main activities for the EXFEL cavity material in the preparation phase (2005-2010) and production phase (2011-2013) are described below.

\section{Preparation phase}

The following main material issues have been considered in the preparation phase: adjustment of the material specification to EXFEL requirements, application of eddy-current scanning (ECS) to the niobium sheets, qualification of new potential material vendors, and assessment of LG material as a possible option for the EXFEL.

\subsection{Revision of the specifications}

The specifications for the material for the EXFEL [20] are based on the specifications for the so-called TESLA cavity [21], for which material for approximately 100 identical cavities for Free-electron LASer in Hamburg (FLASH) facility has been successfully procured. The dimensions and tolerances of SFP have been taken over, however, some metallurgical requirements were revisited. The main discussed points: possible reduction of the residual resistivity ratio (RRR) from 300 to about 250 , possible increase of the limit for the tantalum content from 500 to $1000-1500$ wt. ppm $(\mu \mathrm{g} / \mathrm{g})$, and toleration of grain size bigger than ASTM 4-5.

Lastly, however, a conservative solution that keeps the FLASH requirements without any significant changes was chosen. The central points of the final version of niobium specifications for the EXFEL can be seen in table 1 .

\subsection{Motivation for ECS of niobium sheets}

For several years ongoing discussion has taken place concerning applying (and the necessity) of the eddycurrent scanning (ECS) on niobium sheets prescribed for the production of half-cells of the cavities, for which there are a number of arguments for and against [22]. The work
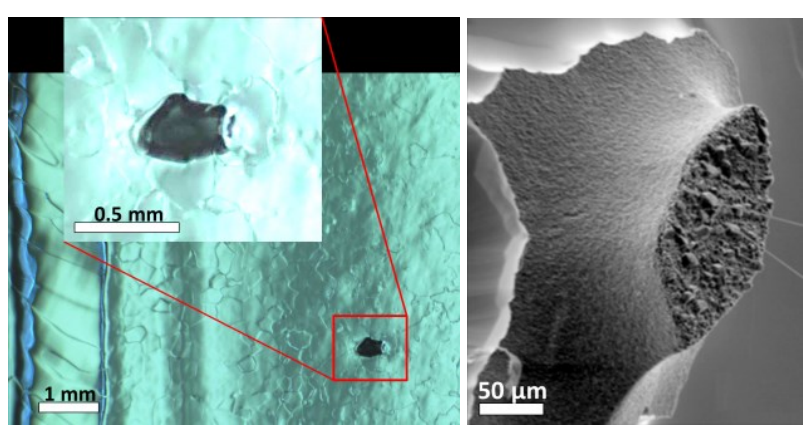

Figure 1. Optical image of the quench area in the cavity Z161 with the marked defect (left) and SEM image (right) of the defect obtained on a sample extracted from the quench area. The protrusion consists of aluminum as identified by EDX and AES analysis.

of reference [23] initiated this activity, by demonstrating that foreign material inclusions in the half-cell can significantly affect cavity performance. Data acquired on samples extracted from some EXFEL prototype cavities confirm this [24, 25]. For example aluminum was detected in one of the EXFEL prototype cavities (figure 1). By monitoring the temperature on the outer cavity surface (T-mapping) during cryogenic RF test of the cavity, it was shown that the inclusion caused thermal breakdown of superconductivity (quench) in this area at 13.7 MV/m. Auger electron spectroscopy (AES) and scanning electron microscopy (SEM) with energydispersive X-ray analysis (EDX) clearly identified aluminum in the hot spot location. The defect was close to the electron-beam welding (EBW) seam and survived the electrochemical polishing (EP) of the cavity. Only two possible reasons can cause such imperfections: either the imbedding happened during niobium sheet production or during mechanical fabrication of the cavity.

In order to reduce the rather significant variation (scattering) in performance of FLASH cavities [26], an ECS device with a special probe for detecting defects in niobium sheets was developed as a remedy against inclusions, and a prototype was built [23, 27]. During scanning of niobium sheets for FLASH, different types of imbedded foreign material inclusions have been detected and the affected sheets were rejected. ECS and optimization of the EBW quality allowed significant

Table 1. Technical specifications of niobium applied to the fabrication of $1.3 \mathrm{GHz}$ superconducting cavities for the EXFEL.

\begin{tabular}{|l|l|l|l|l|l|}
\hline \multicolumn{2}{|l|}{ Electrical and mechanical properties } & \multicolumn{4}{l|}{ Content of the main impurities in wt. ppm $(\mu \mathrm{g} / \mathrm{g})$} \\
\hline $\begin{array}{l}\text { Residual Resistivity Ratio } \\
\text { RRR }\end{array}$ & $>300$ & $\mathrm{Ta}$ & $\leq 500$ & $\mathrm{H}$ & $\leq 2$ \\
\hline Grain size & $\approx 50 \mu \mathrm{m}$ & $\mathrm{W}$ & $\leq 50$ & $\mathrm{O}$ & $\leq 10$ \\
\hline Yield strength, $\mathrm{R}_{\mathrm{p}} 0.2$ & $50<\mathrm{R}_{\mathrm{p}} 0.2<100 \mathrm{~N} / \mathrm{mm}^{2}$ & $\mathrm{Mo}$ & $\leq 50$ & $\mathrm{~N}$ & $\leq 10$ \\
\hline Tensile strength & $>140 \mathrm{~N} / \mathrm{mm}^{2}$ & $\mathrm{Ti}$ & $\leq 50$ & $\mathrm{C}$ & $\leq 10$ \\
\hline Elongation at fracture & $>30 \%$ & $\mathrm{Fe}$ & $\leq 30$ & & \\
\hline Vickers hardness HV10 & $\leq 60$ & $\mathrm{Ni}$ & $\leq 30$ & & \\
\hline
\end{tabular}


improvement of the performance of the cavities for FLASH. This finding is consistent with the results of ECS [28] and systematic tests on different types of niobium sheets [29] which showed, that up to $100 \mu \mathrm{m}$ defects could be resolved in a $100 \mu \mathrm{m}$ depth. Slightly smaller defects can be resolved when closer to, or at the surface.

Supplemental cooperation work of DESY with FNAL has shown that the ECS signal deviation is not always associated with a quench in the detected locations [30]. A single cell cavity was built and tested using one of the sheets with detected deviation of the ECS signal. It turned out that the cavity reached $38 \mathrm{MV} / \mathrm{m}$. Unfortunately, a non-destructive element analysis of the sheets was not performed, what leaves room for speculations.

Experience with semi-finished material procured for the cavities for the upgrade of the Continuous Electron Beam Accelerator Facility (CEBAF) [31] at Jefferson Laboratory (JLAB) supports arguments against ECS [22]. About 1300 sheets (RRR 250) according to JLAB specifications were purchased for $1.5 \mathrm{GHz} 7$-cell cavities, on which no ECS was performed. Yet, there was no report about material defects causing limitation of the required accelerating gradient $\mathrm{E}_{\mathrm{acc}}$ of $19 \mathrm{MV} / \mathrm{m}$ [32].

Despite controversial opinions in the SRF community concerning the ECS, we decided to apply this tool on $100 \%$ of the EXFEL sheets.

According to simulations [33] of the thermal break down (quench at $2 \mathrm{~K}$ ) a local normal-conducting defect of about $100 \mu \mathrm{m}$ size in high purity niobium with RRR 300 causes quench at $\mathrm{E}_{\text {acc }}$ close to $25 \mathrm{MV} / \mathrm{m}$.

Therefore, by eliminating defects with sizes larger than $100 \mu \mathrm{m}$ the requirements of the EXFEL for accelerating gradient $E_{a c c}>23.6 \mathrm{MV} / \mathrm{m}$ should be fulfilled. Detection of defects with sizes larger than $100 \mu \mathrm{m}$ (first of all foreign material inclusions) is, as mentioned above,

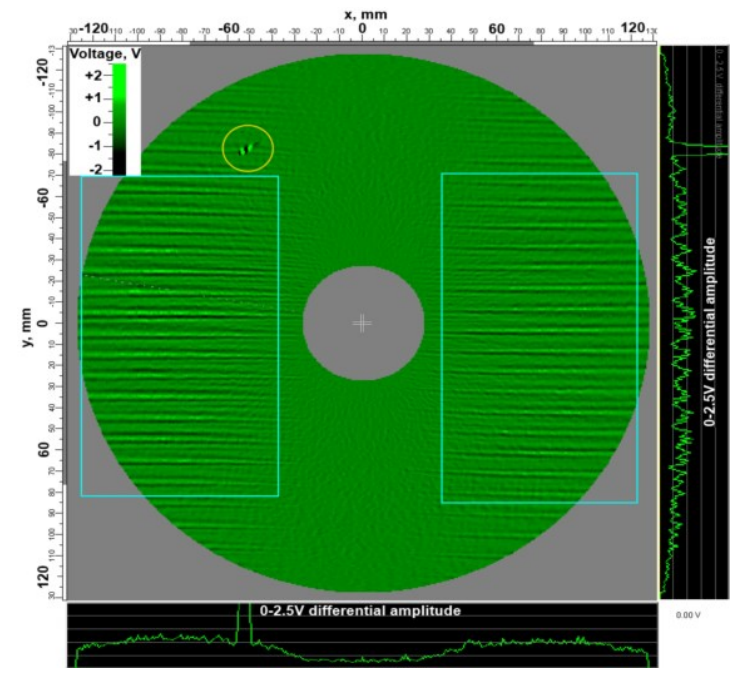

Figure 2. An example of ECS scan result showing a local signal deviation (circle), due to a foreign material inclusion as confirmed later, and a bar pattern of signal deviations (squares) due to surface roughness. a realistic task.

It is frequently difficult to judge the reason for the signal deviation of the ECS. It can be due to inclusions, pores, and flatness or thickness variation of the sheets. An example of the ECS scan result obtained with a device used for the EXFEL production is shown in figure 2. Two types of signal deviations can be seen here. A spot-like one marked by a circle is due to a foreign material inclusion as confirmed later by the material analysis and is expected to be harmful for the cavity performance. The second type is present at almost half of the sheet and is due to the thickness variation and should have no impact on the cavity performance. Thus an elemental analysis of suspicious location is necessary for the final decision.

\subsection{Qualifications of potential vendors}

In relation to material quality for insuring cavity performance, three established niobium suppliers: Wah Chang Corporation (Wah Chang in brief) from USA, Tokyo Denkai Co., Ltd. (Tokyo Denkai in brief) from Japan, and Heraeus Holding GmbH (Heraeus in brief) from Germany have been available for the EXFEL. These companies were earlier qualified for FLASH and the required quality was proven in performance of FLASH cavities.

Furthermore, some additional companies were intended to be qualified for the EXFEL: Companhia Brasileira de Metalurgia e Mineração (CBMM in brief) from Brazil, PLANSEE Composite Materials $\mathrm{GmbH}$ (PLANSEE in brief) from Austria, H.C. Starck GmbH (H.C. Starck in brief) from Germany, Cabot Corporation (Cabot in brief) from USA, Ningxia Orient Tantalum Industry Co., Ltd. (Ningxia OTIC in brief) from China, Northwest Institute for Nonferrous Metal Research (NIN in brief) fom China, and Federalnoe Gosudarstvennoe Unitarnoe Predpriyatie Gosudarstvenny NauchnoIssledovatelsky i Proektny Insitut Redkometallicheskoi Promyshlennosti GIREDMET in cooperation with Institute of Theoretical and Experimental Physics ITEP (ITEP/GIREDMET in brief) from Russia as summarized in table 2 .

For the qualification process of new potential vendors, DESY has defined three steps:

Step 1: Material testing.

Step 2: Single-cell cavity fabrication and treatment followed by RF tests. Single-cell cavities have been produced, treated, and RF tested at DESY in the context of a single-cell program [34].

Step 3: Nine-cell cavity fabrication at the industry and treatment at DESY followed by RF tests.

In Step 1 the material test results had to conform to the requirements of the EXFEL specifications. The RF performance tests within Step 2 and 3 of single-cell and nine-cell cavities after treatment similar to that prescribed for the EXFEL had to fulfil the acceptance criteria of the EXFEL cavities: maximum accelerating gradient $\mathrm{E}_{\mathrm{acc}}$ of at least $26 \mathrm{MV} / \mathrm{m}$ with an unloaded quality factor $Q_{o}$ of at least $1 \times 10^{10}$ and X-ray radiation level lower than $1 \times 10^{-2}$ 
Table 2: Qualification of new niobium vendors for the EXFEL

\begin{tabular}{|c|c|c|c|c|}
\hline Company & $\begin{array}{l}\text { Material } \\
\text { testing }\end{array}$ & $\begin{array}{l}\text { Single-cell } \\
\text { RF tests }\end{array}$ & $\begin{array}{l}\text { Nine-cell } \\
\text { RF tests }\end{array}$ & Remarks \\
\hline CBMM (Brazil) & failed & & & insufficient purity \\
\hline PLANSEE (Austria) & passed & passed & passed & $\begin{array}{l}\text { fabrication of the niobium sheets from ingots } \\
\text { produced at company Heraeus }\end{array}$ \\
\hline $\begin{array}{l}\text { H.C. Starck } \\
\text { (Germany) }\end{array}$ & failed & & & insufficient purity \\
\hline Cabot (USA) & failed & passed & failed & unstable properties \\
\hline $\begin{array}{l}\text { Ningxia OTIC } \\
\text { (China) }\end{array}$ & passed & passed & passed & all steps of niobium production available in-house \\
\hline NIN (China) & passed & failed & & did not deliver sheets for cavities \\
\hline $\begin{array}{l}\text { ITEP/GIREDMET } \\
\text { (Russia) }\end{array}$ & passed & passed & failed & did not establish mass production \\
\hline
\end{tabular}

$\mathrm{mGy} / \mathrm{min}$. The required $26 \mathrm{MV} / \mathrm{m}$ gives $10 \%$ safety margin regarding to $23.6 \mathrm{MV} / \mathrm{m}$ designed gradient for the EXFEL project.

Results of new vendor qualifications are summarized in table 2. The companies CBMM and H.C. Starck failed on the first step of qualification due to insufficient purity of niobium (RRR was below 300). The company NIN did not deliver material for qualification steps 2 and 3. Some details of the qualification process of remaining vendors are mentioned below.

ITEP/GIREDMET (Russia): Russian niobium attracted special interest due to its ultra-high purity. Purification by electrolysis in molten salts using niobiumfluoride with potassium-fluoride (10-20\%) and sodiumfluoride (5-15\%) together with an equimolecular mixture of chlorides $(\mathrm{NaCl}, \mathrm{KCl})$ developed at GIREDMET [35] was applied. This rather expensive refining step before electron beam (EB) melting allows for achieving RRR purity level up to 1000 . Niobium becomes extremely pure in relation to metallic elements. For example tantalum content can be reduced by this treatment from 400 to 3.5 wt. ppm (mg/g).

The Russian company GIREDMET in cooperation with ITEP produced and delivered for evaluation eight niobium sheets (IG-niobium). After proper annealing of the sheets at DESY the measured RRR values were between 667 and 914 with the average value of 743 .

Maximal accelerating gradients of $34 \mathrm{MV} / \mathrm{m}$ have been achieved [36]. The cavities demonstrated a respectable performance, although the ultra-high purity of the IG-niobium did not lead to any spectacular results. Unfortunately, the material for nine-cell cavities was not delivered by the company and an industrial mass production for the EXFEL was not established in Russia.

CABOT (USA): 30 niobium sheets have been delivered to DESY in 2006 for qualification. In spite of the fact that the RRR values of the ingot exceeded the 300 level (330 to 350 ) the RRR of the sheets was mostly below 300 and indicates a lot of scattering between 212 and 307 . Three single cell cavities have been built, tested at DESY and partially at JLAB. Maximum accelerating gradient was $30-34 \mathrm{MV} / \mathrm{m}$ with acceptable $\mathrm{Q}_{0}$.
Nevertheless, according to defined rules the qualification failed because step 1 was not passed. This decision was taken not only on basis of formalities; past experiences also did not speak in favor of Cabot niobium. 60 pieces of niobium sheets have been purchased and used for 9-cell FLASH cavities. For which it turned out that $60 \%$ of the quenches below $25 \mathrm{MV} / \mathrm{m}$ are caused by Cabot sheets. More detailed investigations of niobium sheets for halfcells detected significant purity variation within the sheet and local purity insufficiency (sometimes RRR less than 110).

Finally only two new vendors i.e. PLANSEE from Austria and Ningxia OTIC from China successfully passed all the steps of the qualification and were considered in the procurement procedure as qualified material suppliers for the EXFEL.

Ningxia OTIC (China): first contact with colleagues of the company was in 2003. The company started with niobium of RRR of 100 to 130 . However, they renewed rather quickly their EB melting equipment and since 2006 produced stably high-purity niobium with RRR more than 300 fulfilling the EXFEL specifications. Excellent RF performance with $E_{a c c}$ up to $40 \mathrm{MV} / \mathrm{m}$ was reached on single- and nine-cell cavities produced from Ningxia OTIC niobium sheets [37].

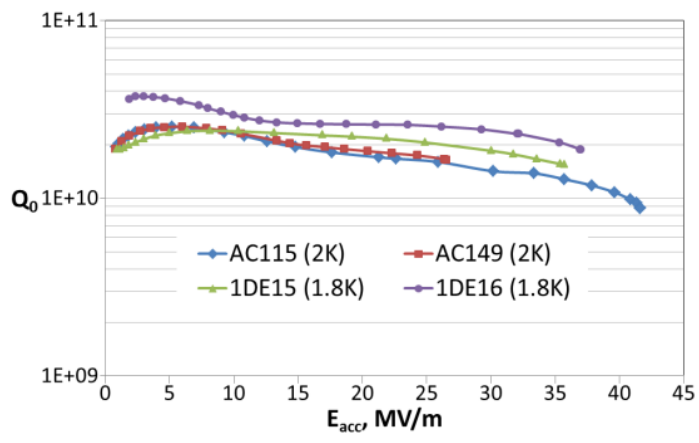

Figure 3. RF performance at $2 \mathrm{~K}$ of the single- (1DE15 and 1DE16) and nine-cell (AC115 and AC149) cavities made of PLANSEE material for qualification of the company. 
PLANSEE (Austria): the company PLANSEE proposed to manufacture niobium sheets for the EXFEL from the ingots EB melted at the company Heraeus. Due to the fact that Heraeus was already qualified, only the transition from ingot to the sheet without purity degradation was required for qualification of PLANSEE. $Q_{o}\left(E_{a c c}\right)$ data of single- and nine-cell cavities confirming the qualification can be seen in figure 3 .

The following sequence of the surface preparation of the single-cell cavities was applied: about $80 \mu \mathrm{m}$ Buffered Chemical Polishing (BCP), $800^{\circ} \mathrm{C}$ annealing for $2 \mathrm{~h}$ in UHV, about $100 \mu \mathrm{m}$ EP, High Pressure Water Rinsing (HPR), and $120^{\circ} \mathrm{C}$ bake for $48 \mathrm{~h}$. Preparation of nine-cell cavities included $110 \mu \mathrm{m} \mathrm{EP}, 800^{\circ} \mathrm{C}$ annealing for $2 \mathrm{~h}, 40$ $\mu \mathrm{m}$ final EP, HPR, and $120^{\circ} \mathrm{C}$ bake for $48 \mathrm{~h}$.

The second type of company qualification was insuring that the material allows fabricate cavity according the European PED 97/23/EC. For that, the vendors have to be certified as producers of material for pressure-bearing parts. This certification was supplementary done by the "notified body" (TUEV Nord Systems $\mathrm{GmbH}$ ) in cooperation with DESY according to the PED [18]. After the examination of the quality management system at the companies, certificates have been granted to them.

In addition, the PED required also qualification of the SFPs used for the pressure-bearing sub-components. Testing of the cavity materials, and creation of corresponding particular material appraisals (PMAs) was done by the "notified body" in cooperation with DESY. Traceability for the pressure-bearing parts is guaranteed by marking and data collection.

\subsection{Large grain cavities}

Manufacture of superconducting cavities by slicing the discs from the melted ingot and producing them by deep drawing and electron beam welding (LG cavities) was considered as an option for the EXFEL [38]. Several single- and eleven nine-cell cavities have been produced at DESY from LG material developed at the company Heraeus.

The cavities demonstrated adequate RF performance satisfying the EXFEL requirements. Even after the somewhat simple BCP treatment procedure consisting of removal of $100 \mu \mathrm{m}$ surface layer inside and $20 \mu \mathrm{m}$ outside, annealing at $800^{\circ} \mathrm{C}$ for $2 \mathrm{~h}$, final BCP of $20 \mu \mathrm{m}$ inside, followed by baking at 125 to $135^{\circ} \mathrm{C}$ for $48 \mathrm{~h}$, accelerating gradients of 25 to $30 \mathrm{MV} / \mathrm{m}$ were achieved in a stable and reproducible manner for both single- and all eleven nine-cell cavities.

After additional EP treatment, including the main EP of about 50 to $70 \mu \mathrm{m}$, followed by an ethanol rinse and $800^{\circ} \mathrm{C}$ annealing for $2 \mathrm{~h}$ and a final EP of about $50 \mu \mathrm{m}$ removal, ethanol rinse, six HPR cycles, and baking at $120^{\circ} \mathrm{C}$, the performance of the cavities was significantly improved up to between 31 and $45.5 \mathrm{MV} / \mathrm{m}$ maximum gradient. The limitation was mostly caused by quenches [39].
Another interesting aspect of LG cavity behavior that has to be stressed is a quite high unloaded quality factor $\mathrm{Q}_{0}$ after EP treatment. $\mathrm{Q}_{\mathrm{o}}$ value for EP treated LG cavities is about 25 to $30 \%$ higher than of the conventional FG cavities [38]. This demonstrates the high potential of $L G$ cavities for applications requiring high $\mathrm{Q}_{0}$ e.g. operating in continuous wave $(\mathrm{CW})$ mode.

It turned out, however, that a shortage on the market of the required quantity of LG material for the EXFEL cannot be unfortunately excluded. In order to minimize the risk for the project it was decided to use conventional FG material for the EXFEL. Finally, one cryogenic module XM-3 consisting of seven LG and one FG cavities was produced and fulfills the EXFEL requirements. The increased value of $\mathrm{Q}_{0}$ factor of $\mathrm{LG}$ cavities was confirmed in the module tests, too.

\section{Production phase}

Material for the series cavities has been ordered in January 2011 by four companies qualified for the EXFEL: Heraeus, Tokyo Denkai, Ningxia OTIC and PLANSEE. Material for the pre-series, foreseen for production of first 32 cavities (dummy, reference, and pre-series cavities), was purchased previously by companies PLANSEE and Heraeus.

Besides all the commercial activities, the material procurement process included also an incoming QC, ECS inspection of the sheets, material analysis to check the conformity with the specifications (analysis of RRR, interstitial and metallic impurities, metallography, tensile test, hardness test, dimensional and surface roughness check), documentation using an engineering data management system (EDMS) at DESY, definition of numbering system and marking, and delivery of the

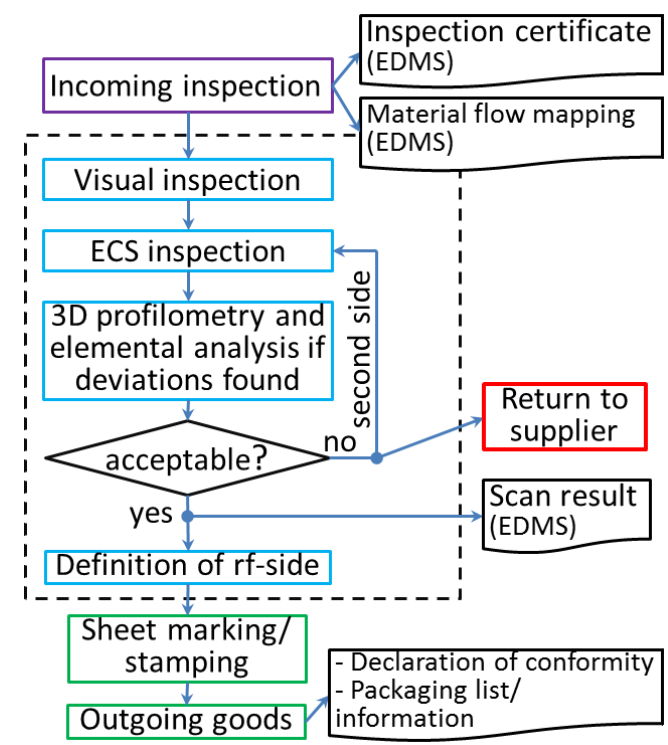

Figure 4. Simplified work flow of the incoming inspection and QC procedure at DESY for the niobium sheets for half-cells fabrication. 
material to companies producing the cavities.

Twelve different types of about 25,000 pieces of SFPs (sheets, plates, tubes, and rods) needed for the EXFEL cavities can be divided in two groups [40]:

1) SFPs to be used for the production of pressure-bearing parts of the cavities according to the PED 97/23/EC, i.e. components being in contact with the liquid helium during operation.

2) SFPs to be used for the production of cavity parts that are not connected to the pressure loaded areas.

The use of the EDMS at DESY [41] has been qualified successfully by the "notified body" according to the PED. EDMS guarantees a world wide access to the documents and makes it possible to check the current status and location of each SFP.

An example of the work flow for the niobium sheets for half-cells, the most critical procedure, is shown in figure 4, and also its connection to the process of the EDMS

Sheets that passed visual inspection have been ECSscanned as described in the following section.

The sheets suspected for foreign material inclusions have been thoroughly examined non-destructively.

Close cooperation with the companies significantly contributed to the high quality of the material. Thanks to well-timed feedback to the companies, the quality of the material improves from shipment to shipment. At the outset occurrence of defects was close to $10 \%$, whereas after few shipments it was reduced by an order of magnitude and remains close to constant for each company.

The relatively low number of cavities, about $7 \%$, limited by quench below $20 \mathrm{MV} / \mathrm{m}$ (which can be caused by material and other reasons such as EBW problems) as fabricated till now for the EXFEL allows us to conclude that the quality of procured material is high, i.e. the QC and joint work with companies is quite efficient.

\subsection{Scanning statistics for the sheets}

Two new eddy current devices were developed and built

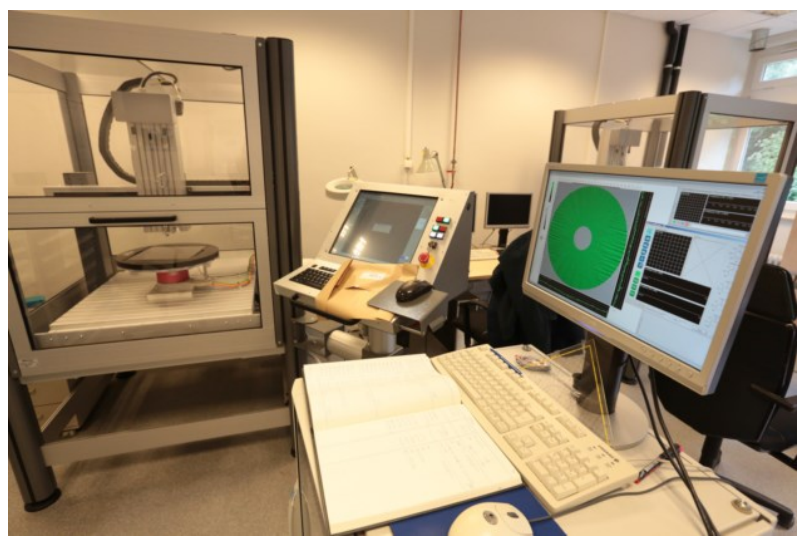

Figure 5. Device for ECS of niobium sheets (scanning head on the left and control computer on the right). in conjunction with the industry for scanning of EXFEL niobium sheets (figure 5). A circular coil generates homogeneous eddy currents in the niobium sheet, whereas inhomogeneities like holes or inclusions of materials, having conductivity different from that of niobium, lead to a distortion of the eddy current flow. This results in a change of the eddy current field, which will be detected as a signal deviation by a pick-up coil specially developed for niobium.

As mentioned above, all EXFEL sheets, including those replaced and reprocessed by the suppliers, were scanned [42].

The operator judges whether a sheet is qualified by means of following criteria:

- first scanned side good, sheet is qualified;

- first scanned side bad, second scanned side good, sheet is qualified;

- first and second scanned sides bad, sheet is not

qualified and belongs to non-usable sheets.

Subsequent non-destructive investigation of nonusable sheets includes visual surface inspection, optical microscopy and 3D profilometry, surface roughness measurement, and X-ray fluorescence elemental analysis. The aim of these additional analyses was to separate the sheets for rejection from the sheets suitable for reconditioning.

Detailed analysis of the non-qualified sheets allowed us to define two main categories of defects: foreign material inclusions and topographical defects (scratches, holes, pits, marks, and delamination).

Non-qualified sheets have been returned to the vendors. All sheets with foreign material inclusions have been replaced. The sheets with topographical defects have been mostly reconditioned (re-grinded) by vendors and accepted after repeated QC. Delaminations were revealed on several sheets at the beginning of the production. The cause of this was discovered by the companies and the number of sheets with delaminations was radically reduced.

Figure 6 represents statistical data from the ECS of the niobium sheets. Approximately $26 \%$ of the sheets have been scanned on both sides. About $2 \%$ (310 out of 15612) of all scanned sheets were classified as unusable.

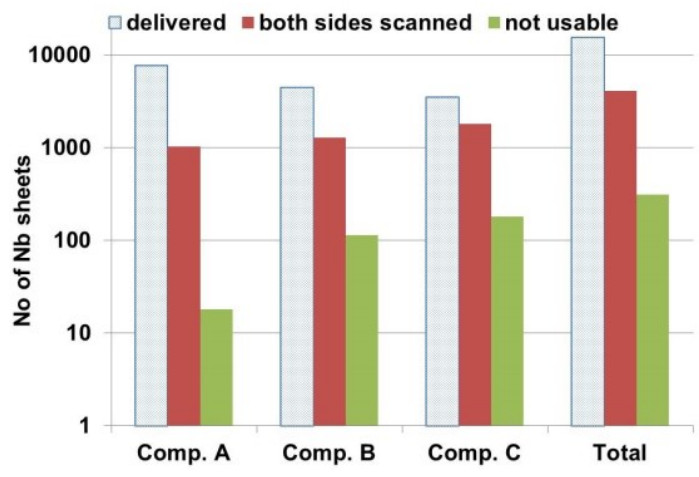

Figure 6. Statistics from ECS of niobium sheets. 

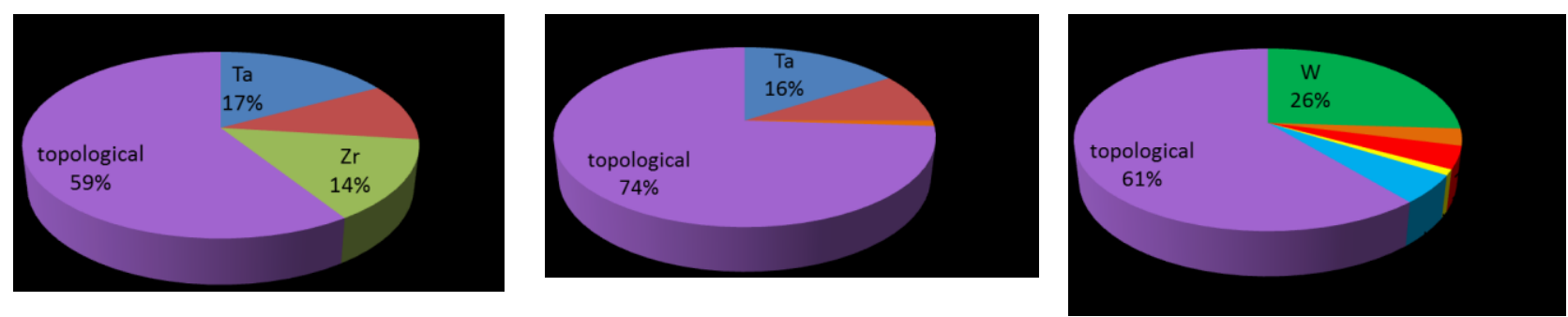

Figure 7. Distribution of imperfections in niobium sheets for the three vendors A, B, and C respectively.

Quality distinctions between the three suppliers of niobium material (A, B, and C) can be clearly seen.

The percentage distribution of imperfections found by the ECS and non-destructive analysis of the material from the three suppliers is shown in figure 7.

In the sheets from all three suppliers inclusions of steal $(\mathrm{Fe}, \mathrm{Ni}, \mathrm{Cr}$ or $\mathrm{Fe}, \mathrm{Ni}, \mathrm{Co})$ were detected. Inclusions of tantalum were found in the sheets from company A and $\mathrm{B}$; titanium from companies $\mathrm{B}$ and $\mathrm{C}$; zirconium from the company $\mathrm{A}$; tungsten, molybdenum, and zinc from company $\mathrm{C}$.

\section{Influence of the EP on foreign material inclusions in niobium}

The main treatment procedure of the inside surface of the cavities for the EXFEL is EP. A layer 110 to $140 \mu \mathrm{m}$ thickness has to be removed $[20,43]$. In connection to this a justified question may arise; whether the foreign material inclusion will disappear during EP, or shall remain and possibly cause a quench in the cavity. This subject was checked on some rejected sheets. Samples of round shape with defects in the middle area have been extracted from the sheets and polished applying equipment as described in [44]. The EP parameters are similar to those used for the EXFEL cavities. Detailed analysis of the evolution of the surface and influence of the EP process on the inclusions has been performed. It includes optical microscopy with VHX-5000 Keyence digital microscope, SEM, and EDX analysis.

Several results illustrating the effect of the EP on defects can be seen in figures 8 and 9 . The investigations showed that some foreign material inclusions are almost unaffected by the EP, and other disappeared completely,
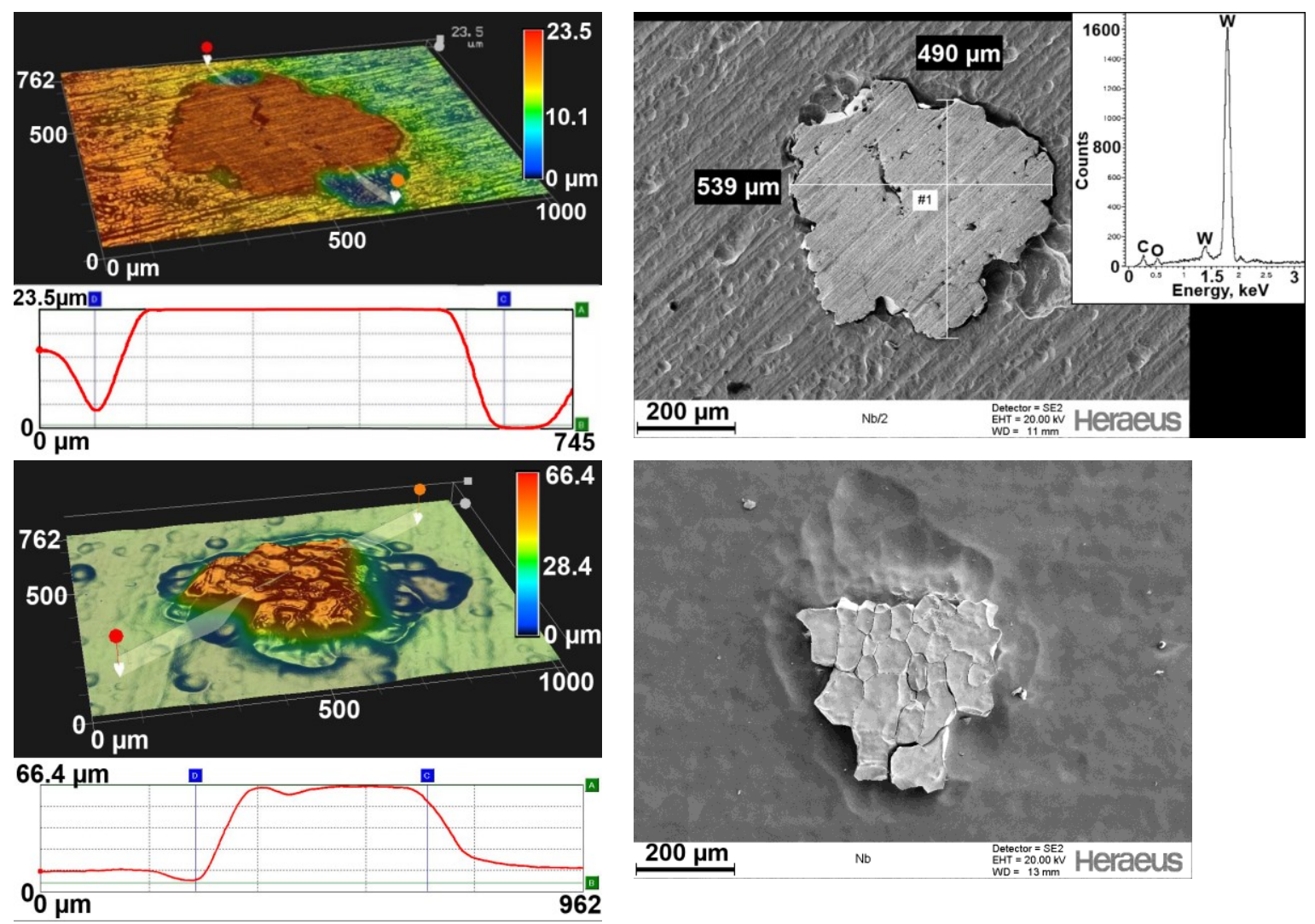

Figure 8. Optical (left) and SEM (right) microscopy of tungsten inclusion (as identified by EDX, see a part of the EDX spectra in the inset) found in the area indicated by the ECS and comparison of the inclusion before (top row) and after (bottom row) $150 \mu \mathrm{m}$ EP. Note different orientation of the images. 

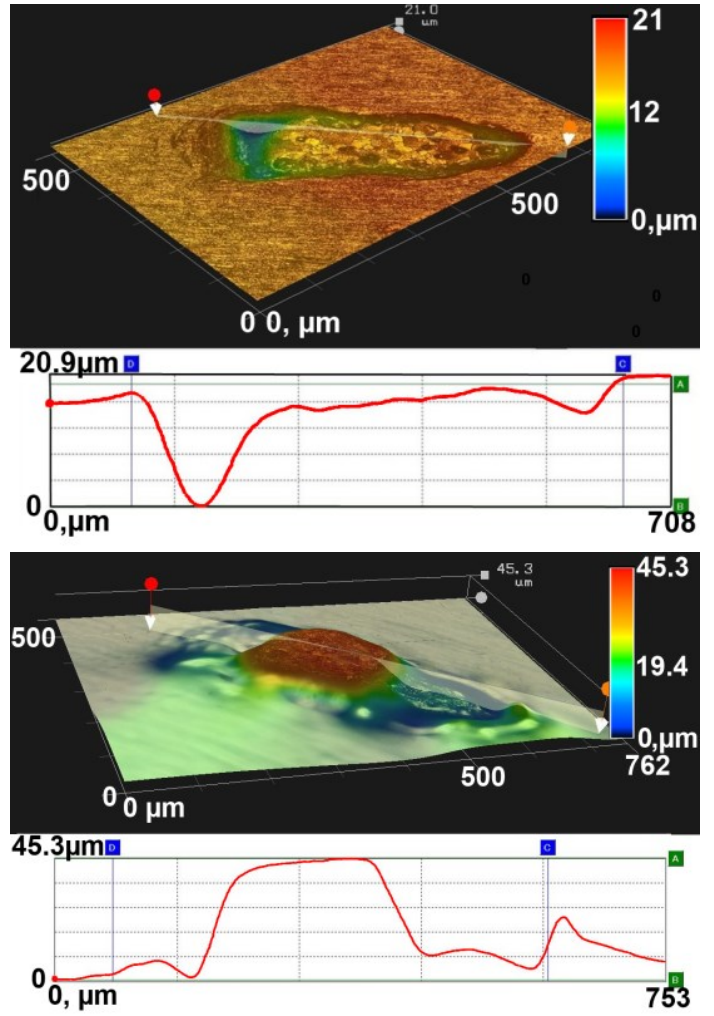
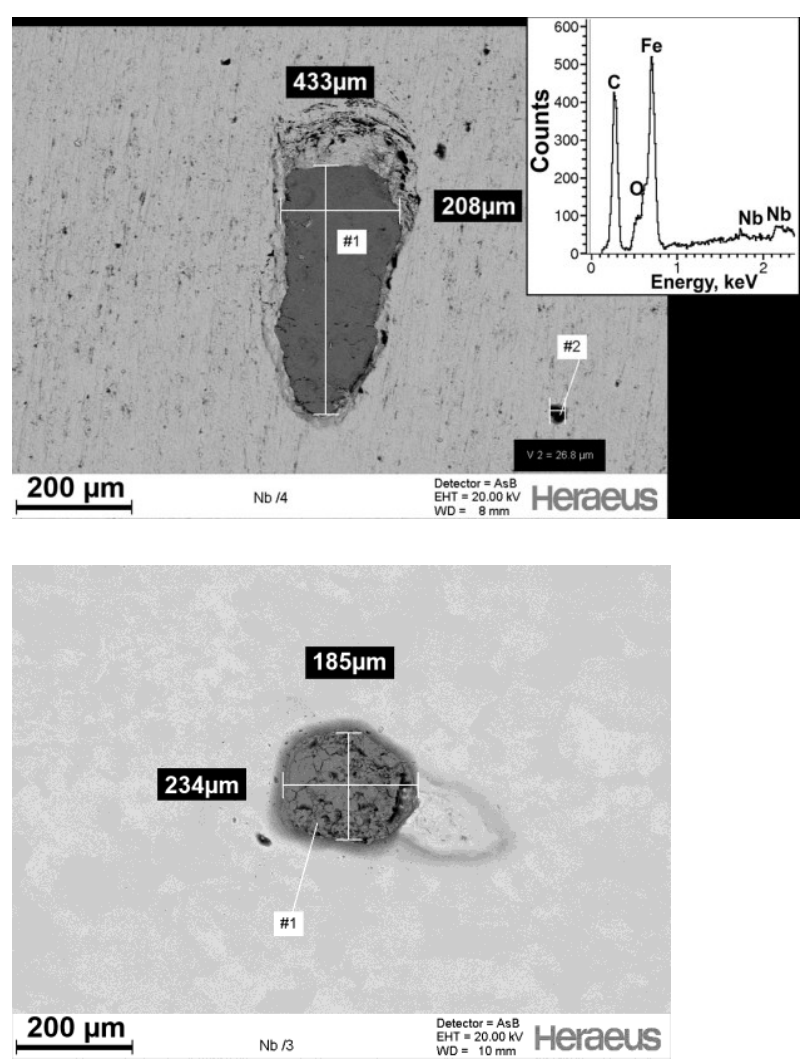

Figure 9. Optical (left) and SEM (right) microscopy of iron inclusion (as identified by EDX, see a part of the EDX spectra in the inset) found in the area indicated by the ECS and comparison of the inclusion before (top row) and after (bottom row) $150 \mu \mathrm{m}$ EP. Note different orientation of the images.

with results summarized in table 3 . Clearly it depends on the inclusion size, as well as on the material and its concentration, since the EP removal rate is different for different elements.

It is well known (e.g. $[3,45])$ that BCP and EP work similarly for niobium. In both cases transformation of niobium to $\mathrm{Nb}^{5+}$ and creation of niobium pentoxide $\mathrm{Nb}_{2} \mathrm{O}_{5}$ takes place. In the first case nitric acid $\mathrm{HNO}_{3}$ plays the role of oxidant whereas, in the second case the oxidation is reached by $\mathrm{SO}_{4}{ }^{2-}$ ions from sulphuric acid $\mathrm{H}_{2} \mathrm{SO}_{4}$ at applied electrical field. $\mathrm{HF}$ decomposes the oxides into soluble $\mathrm{NbF}_{5}$ or $\mathrm{H}_{2} \mathrm{NbOF}_{5}$ that moves into the mixed acid solution. The effect of $\mathrm{BCP}$ and $\mathrm{EP}$ on the foreign material inclusions is different. BCP either dissolves the inclusion or removes the niobium around the inclusion finally eradicating it if the removed niobium

Table 3. Influence of EP on different foreign material inclusions in the niobium sheets

\begin{tabular}{|c|c|c|c|c|}
\hline $\begin{array}{c}\text { Sheet } \\
\text { No. }\end{array}$ & $\begin{array}{c}\text { Sheet } \\
\text { side }\end{array}$ & $\begin{array}{c}\text { Material } \\
\text { of } \\
\text { inclusion }\end{array}$ & $\begin{array}{c}\text { Size of the } \\
\text { inclusions, } \\
\mu \mathrm{m}^{2}\end{array}$ & $\begin{array}{c}\text { Defect after } \\
\text { about } 150 \\
\mu \mathrm{m} \mathrm{EP}\end{array}$ \\
\hline 374 & $\mathrm{~S} 1$ & $\mathrm{Ta}$ & $280 \times 500$ & remains \\
\hline 449 & $\mathrm{~S} 2$ & $\mathrm{Zr}$ & $270 \times 280$ & vanished \\
\hline 5003 & $\mathrm{~S} 1$ & $\mathrm{Fe}$ & $210 \times 430$ & remains \\
\hline 5021 & $\mathrm{~S} 2$ & $\mathrm{~W}$ & $490 \times 540$ & remains \\
\hline 4777 & $\mathrm{~S} 1$ & $\mathrm{Ti}$ & $360 \times 470$ & vanished \\
\hline
\end{tabular}

layer is sufficient. Influence of the EP on foreign material inclusions is completely different depending on the type of defect and its individual properties. The applied voltage for example is defined not only by anode and cathode potentials but also by the voltage drops in the conductors and contacts that will not be the same for inclusions and niobium. As a result, instead of being removed by polishing, the pitting or gas evolving process can still take place. An active dissolution, when no viscous layer is formed and the metallic ions dissolve freely in electrolyte, or even passivation with a formation of a stable insoluble film preventing further corrosion can appear. Thus, it is no wonder that after EP in some cases we see a pronounced protrusion indicating no influence of the EP on the inclusions.

\section{Indications of foreign material inclusions in cavities}

From more than 650 of the cavities fabricated for the EXFEL up to now, only few cavities have been discovered with strong indications of presence of foreign material inclusions on the inner surface. Figure 10, 11, and 12 represent defects found on the inner surface of cavities $\# 1, \# 2$, and $\# 3$. The defects look very similar to the inclusions observed on niobium sheets and extracted samples. 

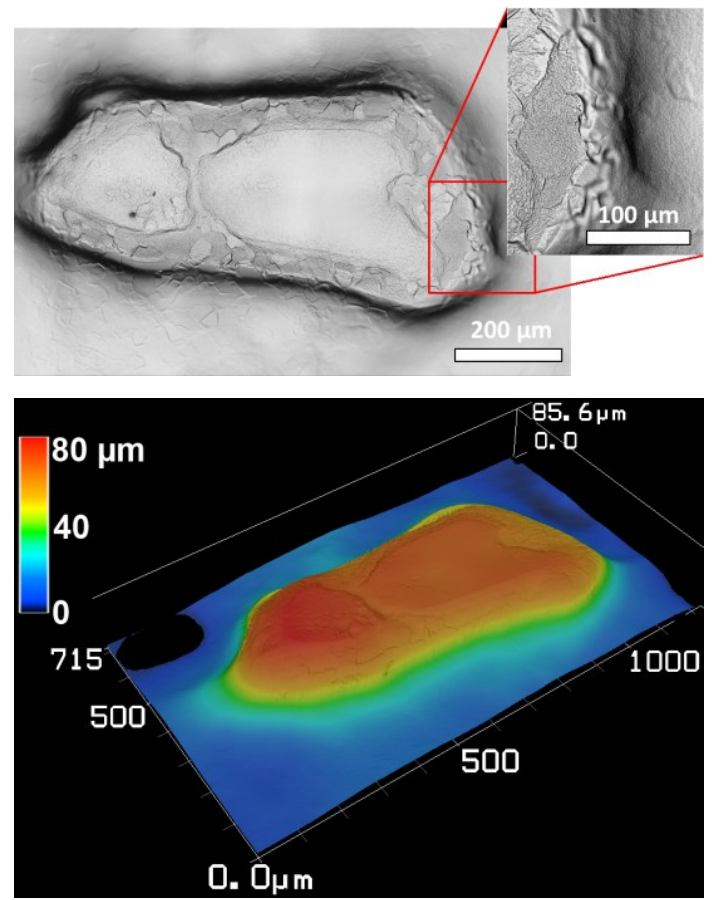

Figure 10. Morphology (top) and 3D view (bottom) of a defect observed on cavity \#1 and visualized by laser scanning microscopy on replica sample taken by producer. Defect has a protrusion-like shape of about 120 $\mu \mathrm{m}$ height with a flat top.
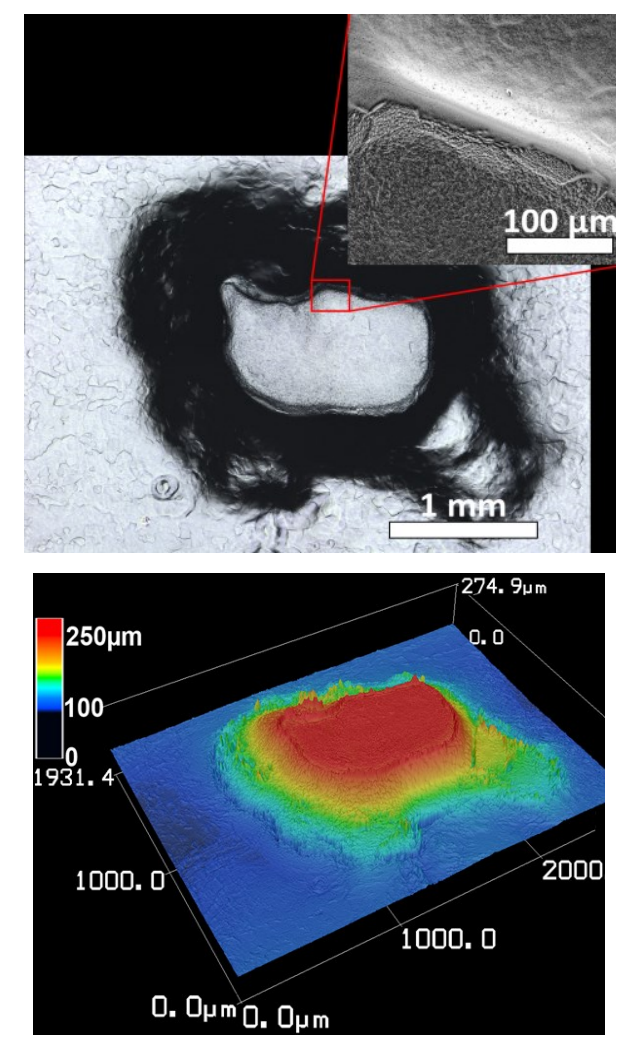

Figure 11. Morphology (top) and 3D view (bottom) of a defect observed on cavity \#2 and visualized by laser scanning microscopy on replica sample taken by producer. The height of the protrusion is about $140 \mu \mathrm{m}$.

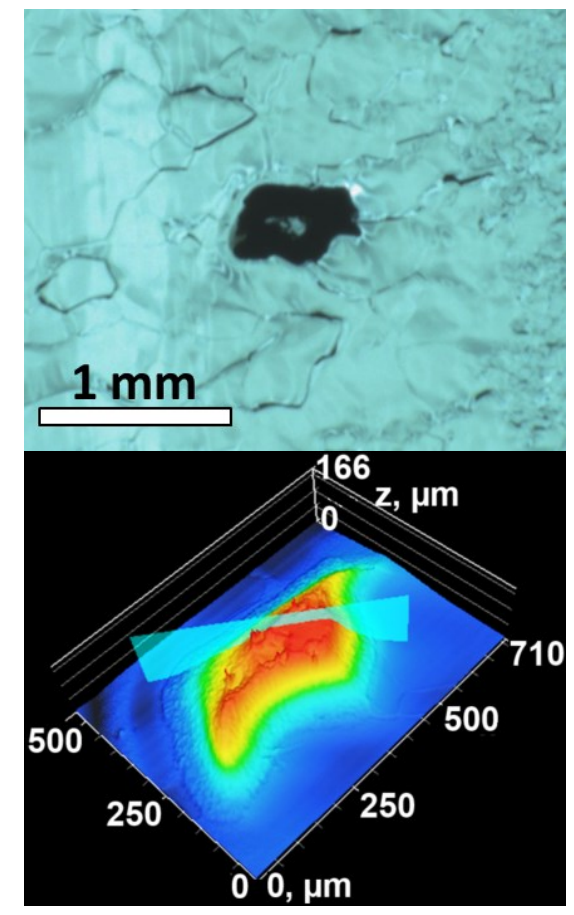

Figure 12. Defect observed by the high resolution OBACHT tool (top) on cavity \#3 and visualized by laser scanning microscopy on replica sample (bottom). The height of the protrusion is about $130 \mu \mathrm{m}$.

Defects on cavities \#1 and \#2 were revealed during optical inspection by the manufacturer. One defect has been found near the iris and another one in the beam tube.

The cavities were sorted out for additional investigations and finally for local grinding, but were not RF tested yet. The defects illustrate clearly pronounced protrusion of 120 to $140 \mu \mathrm{m}$ and were not affected by the main EP treatment as revealed by laser scanning microscopy on replica [46] samples. This indicates different chemical properties of the defects than of the niobium. Additionally, the surface microstructure of the inclusions is completely different than of the niobium surface (see inset of figures 10 and 11). These facts are considered as strong hints for foreign material inclusions. Similar morphology difference has been observed on defects with foreign material inclusions confirmed by SEM and EDX material analysis.

A similar defect on the cavity \#3 (figure 12) caused a limitation of the cavity performance at 16 $\mathrm{MV} / \mathrm{m}$ by quench without any radiation, and was later observed by the high resolution OBACHT tool [47] and visualized by laser scanning microscopy on replica sample. A surface flaw, found close to the equator, also looks like a foreign material inclusion. Images of the defect in cavity \#3 are very similar to images shown in figure 1 . The dimensions of the defect are about $0.5 \mathrm{x}$ $0.7 \mathrm{~mm}^{2}$ with $130 \mu \mathrm{m}$ heights. It is difficult to imagine that the described inclusions were present in the original 
niobium material. Such big defects of more than $500 \mu \mathrm{m}$ size would be definitely detected by the ECS as discussed in section 2.2. It can be supposed that the inclusions were embedded during one of the production steps like deep drawing, clamping for machining, or assembly for EBW.

Presented data regarding foreign material inclusions in cavities stress the necessity of the inclusion avoidance in the original niobium material.

\section{Summary}

In the preparation phase some new companies have been qualified as potential material suppliers for the EXFEL cavities. Together with already experienced companies, the required amount of cavity material on the market for the EXFEL was insured.

11 prototype LG cavities were produced and RF tested as a possible option for the EXFEL. Encouraging results in $Q_{o}$ and $E_{a c c}$ have been reached. One cryogenic module XM-3 with LG cavities has been successfully built. Finally, it was decided that fine grain material would be used for the EXFEL, due to possible material delivery shortage and associated project risk.

DESY has taken over the procurement, QC, documentation, and shipment of material to producers of the EXFEL cavities. Procurement was done according the European PED supported by the "notified body" (TÜV NORD Systems GmbH). Timely feedback to material providers contributed to successful production of cavity material.

After scanning more than 15000 niobium sheets by ECS it was stated that in order to define the RF side, $26 \%$ of the sheets were scanned on both sides. About $2 \%$ of the material was rejected. Most of the ECS signals indicated foreign material inclusions or demonstrated topographical flaws.

It can be concluded that material inspection based on ECS of $100 \%$ of the delivered material, and supplemented by non-destructive detailed investigation like X-ray element analysis and digital microscopy, is a reasonable inspection method to avoid diminished RF performance of cavities caused by material. First of all, it allows better choice of the side of the sheet to be exposed to the RF. Moreover, not utilizing such material inspection would mean that in the worst case mentioned the $2 \%$ sheets rejected will affect the performance of approximately every third cavity, supposing that the unusable sheets would be homogeneously distributed in the production lots. For that it has been assumed that only one of eighteen sheets might negatively impact the performance of the complete cavity.

In order to check the influence of the EP treatment on the inclusions, some samples have been extracted from rejected sheets and investigated. Despite the fact that in few cases the foreign material inclusion disappeared, often the foreign material inclusions remained present even after $150 \mu \mathrm{m}$ of EP removal

Recent experience with industrial production of about 650 EXFEL cavities shows that an inclusion can be imbedded not only during sheet production, as defects indicating foreign material inclusions were detected in EXFEL cavities following their manufacture.

The relatively low number of EXFEL cavities up to now (about 7\%) with hard quenches below $20 \mathrm{MV} / \mathrm{m}$ allows us to conclude a high quality of procured material and cavity fabrication process. The procurement and QC of the material for the EXFEL cavities as well as a joint

\section{Acknowledgements}

Many thanks to the EXFEL cavity material team, especially to A. Ermakov, A. Poerschmann, J. Dammann, L. Schaefer, S. Arnold, M. Lengkeit, D. Klinke, and J. Hermann for engaged efforts. Many thanks to H. Weise, W.-D. Moeller, and D. Reschke for support in our work.

Many thanks to the company Heraeus Holding GmbH for the SEM data received on the EP-samples.

\section{References}

[1] H. Padamsee, J. Knobloch, and T. Hays, RF Superconductivity for Accelerators (Wiley-VCH, Weinheim, Germany, 2008), 2nd ed., p. 521.

[2] H. Padamsee, RF Superconductivity (Wiley-VCH, Weinheim, Germany, 2009), p. 448.

[3] C. Antoine, Materials and surface aspects in the development of SRF niobium cavities, EUCARDCERN Monographs, (Institute of Electronic Systems Warsaw University of Technology, Warsaw, 2012), Vol. 12, p. 106.

[4] W. Singer, SRF Cavity Fabrication and Material, Proceedings of the CAS-CERN Accelerator School: Superconductivity for Accelerators, Erice, Italy, 24 April - 4 May 2013, edited by R. Bailey, CERN2014-005 (CERN, Geneva, 2014), DOI: 10.5170/CERN-2014-005.

[5] Ch. Reece and G. Ciovati, Superconducting RF Technology R\&D for Future Accelerator Applications, Reviews of Accelerator Science and Technology, Vol. 5, (2012) 1-5, p.1-32.

[6] S. Posen, M. Liepe, $\mathrm{Nb}_{3} \mathrm{Sn}-$ Present Status and Potential as an Alternative SRF Material, Proceedings of the 27th International Linear Accelerator Conference LINAC2014, Geneva, Switzerland, August 31-September 5, 2014, TUIOC03, P. 431-436, ISBN 978-3-95450-142-7

[7] M. Krishnan, E. Valderrama, C. James, X. Zhao, J. Spradlin, A-M Valente Feliciano, L. Phillips, C. E. Reece, K. Seo, and Z. H. Sung, Energetic condensation growth of $\mathrm{Nb}$ thin films, Phys. Rev. ST Accel. Beams 15, 032001 (2012), p. 1-15.

[8] A.-M. Valente-Feliciano, HIPIMS: A New Generation of Film Deposition Techniques for SRF Applications, Proceedings of the 16th International Conference on RF Superconductivity, SRF2013, Paris, France, September 23-27, 2013, WEIOA01, p. 754-760, ISBN 978-3-95450-143-4 
[9] A. Gurevich, Appl. Phys. Lett. 88 (2006) 012511.

[10] C. Z. Antoine, J.C. Villegier, and G. Martinet, Appl. Phys. Lett. 102 (10) (2013) 102603.

[11] "ILC Technical Design Report", June 2013, ISBN 978-3-935702-74-4 www.linearcollider.org

[12] "ESS Technical Design Report", April 23, 2013." ESS-doc-274, ISBN 978-91-980173-2-8 http://eval.esss.lu.se/cgibin/public/DocDB/ShowDocument?docid=274

[13] J. N. Galayda, "The Linac Coherent Light Source-II Project", TUOCA01, Proceedings of IPAC 2014, Dresden, Germany (2014).

[14] A. Grassellino, A Romanenko et al. Nitrogen and argon doping of niobium for superconducting radio frequency cavities: a pathway to highly efficient accelerating structures, Supercond. Sci. Technol. 26, (2013), 102001, p. 1-6.

[15] A. Crawford et al. The Joint High Q0 R\&D Program from LCLS-II, Proceedings of the 5th International Particle Accelerator Conference IPAC2014, Dresden, Germany, June 15-20, 2014,P. 2627-2630, WEPRI062, ISBN 978-3-95450-132-8

[16] XFEL Technical Design Report, DESY 2006-097, Hamburg, July 2007, www.xfel.eu

[17] E. Haebel et al., "Cavity Shape Optimization for a Superconducting Linear Collider", Proc. of the 15th Int. Conf. on High Energy Acc. (HEACC '92), vol. 2, Hamburg, Germany, July 1992, p. 957 (1992).

[18] Pressure Equipment Directive 97/23/EC of the European Parliament and of the council, of 29 May $1997 \mathrm{http}: / /$ ec.europa.eu/enterprise/sectors/pressureand-gas/documents/ped/

[19] P. Kneisel, G. Ciovati, P. Dhakal, K. Saito, W. Singer, X. Singer, G. R. Myneni, Review of ingot niobium as a material for superconducting radiofrequency accelerating cavities. Nuclear Instruments and Methods in Physics Research A774 (2015) 133-150

[20] Specification Documents for Production of European XFEL 1.3 GHz SC Cavities. DESY, 2009.

[21] TESLA technical design report, Part II The Accelerator, edited by R. Brinkmann et al., Deutsches Elektronen Synchrotron, Hamburg, March 2001.

[22] 16th International Workshop on RF Superconductivity SRF2013, September 23-27, 2013, Paris, France. Hot topic discussion.

[23] W. Singer, D. Proch, and A. Brinkmann, Diagnostics of defects in high purity niobium. Particle Accelerators, 1998, Vol. 60, pp. 83-102.

[24] X. Singer, S. Aderhold, A. Ermakov, D. Reschke, W. Singer, K. Twarowski, M. Hoss, D. Watzal, Investigation of samples separated from prototype cavities of the European XFEL, THPO055, 15th International Conference on RF Superconductivity (SRF2011), Chicago, July 25-29, USA (2011).

[25] W. Singer, X. Singer, S. Aderhold, A. Ermakov, K. Twarowski, R. Crooks, M. Hoss, F. Schölz, and B. Spaniol. Surface investigation on prototype cavities for the European X-ray Free Electron Laser. Phys. Rev. ST Accelerators and Beams 14, 050702 (2011).

[26] B. Aune et al. The superconducting TESLA Cavities. DESY 00-031, February 2000. ISSN 0418-9833

[27] W. Singer, A. Brinkmann, D. Proch, X. Singer, Quality requirements and control of high purity niobium for superconducting RF cavities. Physica C 386 (2003) 379-384.

[28] C. Boffo et al., "Eddy current scanning at Fermilab," in Proceedings of the RF Superconductivity Workshop 2005, Ithaca, NY, USA, Jul. 2005.

[29] C. Boffo, P. Bauer, M. Foley, C. Antoine, C. Cooper, and A. Brinkmann, "Eddy Current Scanning of Niobium for SRF Cavities at Fermilab", IEEE TRANSACTIONS ON APPLIED SUPERCONDUCTIVITY, VOL. 17, NO. 2, JUNE 2007.

[30] G. Wu, L. Cooley, D. Sergatskov, J. Ozelis1, A. Brinkmann, W. Singer, X. Singer and M. Pekeler. The RF performance of cavity made from defective niobium material determined by Eddy Current Scanning. Applied Superconductivity, IEEE, Vol.21, Issue 3, p. 1900 - 1902, 2011

[31] J. Sekutowicz et al.; “Cavities for JLab's $12 \mathrm{GeV}$ Upgrade", PAC 2003, paper TPAB085.

[32] F. Marhauser, A. Burrill, K. Davis, D. Forehand, C. Grenoble, J. Hogan, R. Overton, A. Reilly, R.A. Rimmer, M. Stirbet, "Fabrication and testing status of CEBAF $12 \mathrm{GeV}$ upgrade cavities", Proceedings of IPAC2011, San Sebastián, Spain MOPC112.

[33] D. Reschke. Thermal model calculations for 1.3 GHz TTF accelerator cavities. Proceedings of 8th Workshop on RF Superconductivity, Abano Terme (Padova), Italy, October 6-10, 1997, pp. 385-396.

[34] A. Schmidt, A. Brinkmann, J. Iversen, A. Matheisen, D. Reschke, M. Schäfer, W. Singer, V. Sousa, J. Tiessen, D. Vermeulen. 1.3 GHz niobium single-cell fabrication sequence. TTC Report 201001. pp. 1-11.

[35] L. Voronenko, A. Eljutin, K. Kovalev and F. Kovalev. Verfahren zur Hochreinniobproduction. Patent C 22 B 34/24 (DE $101 \quad 12822$ A 1 ), 11.10.2001.

[36] D. Reschke, A. Brinkmann, J. Iversen, W. Singer, J. Ziegler, ,Status of the XFEL testcavity programm“, Proceedings of LINAC 2006, Knoxville, Tennessee USA, TUP026.

[37] A. Brinkmann, J. Iversen, D. Reschke, W. Singer, X. Singer, K. Twarowski, J. Ziegler, "Progress of the test cavity program for the European XFEL", Proceedings of the 13th International Workshop on RF Superconductivity, Peking University, Beijing, China, TUP74 (2007).

[38] W. Singer, S. Aderhold, A. Ermakov, J. Iversen, D. Kostin, G. Kreps, A. Matheisen, W.-D. Moller, D. Reschke, X. Singer, K. Twarowski, H. Weise, H.-G. Brokmeier. Development of large grain cavities. Phys. Rev. STAB 16 (2013), 012003-1. 
[39] D. Reschke, S. Aderhold, A. Goessel, J. Iversen, S. Karstensen, D. Kostin, G. Kreps, A. Matheisen, W.D. Möller, F. Schlander, W. Singer, X. Singer, N. Steinhau-Kühl, A. Sulimov, K. Twarowski, "Results on large grain nine-cell cavities at DESY: gradients up to $45 \mathrm{MV} / \mathrm{m}$ after electropolishing", Proceedings of SRF2011, Chicago, IL USA, TUPO046 (2011).

[40] J. Iversen, A. Brinkmann, J. Dammann, L. Hagge, W.-D. Moeller, A. Poerschmann, W. Singer, X. Singer, "Supply of $\mathrm{Nb} / \mathrm{NbTi}$ semi-finished products for series SC cavity fabrication for the European XFEL, quality control, logistics and documentation", XXVI Linear Accelerator Conference (LINAC 2012), September 9-14, TelAviv, Israel, MOPB058 (2012).

[41] J. Bürger et al., Towards industrialization: supporting the manufacturing process of superconducting cavities at DESY, Phys. C, 44, (2006), pp. 268-271

[42] A. Brinkmann, S. Arnold, A. Ermakov, J. Iversen, M. Lengkeit, A. Pörschmann, L. Schäfer, W. Singer, X. Singer. Statistic to Eddy-Current Scanning of niobium sheets for the European XFEL. Proceedings of the 16th International Workshop on RF Superconductivity SRF2013, September 23-27, 2013, Paris, France, MOP032

[43] W. Singer et al., Preparation phase for $1.3 \mathrm{GHz}$ cavity production of the European XFEL, IPAC2010, Kyoto, Japan, May 23-28, THOARA02
[44] P. Michelato, L. Monaco. Evolution of the defects on the niobium surface during $\mathrm{BCP}$ and $\mathrm{EP}$ treatments. Proceedings of the 14th International Conference on RF Superconductivity SRF2009, September 20-29, 2009, Berlin-Dresden, Germany, THPPO091.

[45] V. Palmieri. Fundamentals of electrochemistry-the electrolytic polishing of metals: application to copper and niobium. Proceedings of the 11 th Workshop on RF Superconductivity SRF 2003, September 8-12, 2003, Lübeck/Travemünde, Germany. WET02.

[46] A. Navitski, E. Elsen, B. Foster, R. Laasch, D. Reschke, J. Schaffran, W. Singer, X. Singer, Y. Tamashevich, "R\&D on cavity treatments at DESY towards the ILC performance goal," Proceedings of 16th International Conference on RF Superconductivity, SRF 2013, Sep. 23-27, Paris, France, MOP053, p. 240-243, ISBN 978-3-95450143-4 (2013).

[47] A. Navitski, A. Prudnikava, Y. Tamashevich, "Progress of R\&D on SRF cavities at DESY towards ILC performance goal," Proceedings of the 5th International Particle Accelerator Conference IPAC'14, June 15-20, Dresden, Germany, WEPRI011, p. 2499- 2501, ISBN 978-3-95450-1328 (2014). 